\title{
A Reduction in Stimulus Duration can Improve Wavelength Discriminations Mediated by Short-wave Cones
}

\author{
J. D. MOLLON, ${ }^{*} \ddagger$ SUSAN ASTELL, ${ }^{*}$ C. R. CAVONIUS $\dagger$
}

Received 4 April 1991

\begin{abstract}
Virtually all visual discriminations become less accurate when either the luminance or the duration of the stimulus is reduced. An exception is found for wavelength discriminations near $\mathbf{4 6 0} \mathbf{~ n m}$, where an increase in either luminance or duration can cause the threshold to rise. For flashes of $100 \mathrm{msec}$ or less, the critical variable is the total energy of the flash (i.e. the product of retinal illuminance and flash duration), and wavelength discrimination is optimal at an intermediate value; higher stimulus energy causes discrimination to deteriorate. To explain these findings we suppose that discrimination in this region of the spectrum is mediated by a channel that draws opposed signals from the short-wavelength cones and from some combination of the middle- and long-wavelength cones, and that high stimulus energies cause saturation of this channel.
\end{abstract}

Colour vision Wavelength discrimination Stimulus duration Short-wavelength cones Tritanopia

\section{INTRODUCTION}

Most sensory discriminations deteriorate when the stimulus is made very brief. Auditory discrimination of frequency offers a classical example: the threshold for discriminating the pitches of two tones becomes greater as duration is reduced below $200 \mathrm{mscc}$ (c.g. Moorc, 1973). In the case of vision, analogous results are found not only for the simple detection and discrimination of luminance (Graham \& Kemp, 1938), but also for a variety of other functions, such as vernier acuity (Foley \& Tyler, 1976), judgement of orientation (Andrews, 1967), shape constancy (Leibowitz, Mitchell \& Angrist, 1954), discrimination of spatial phase (Nyman, Laurinen \& Campbell, 1986; Tyler \& Gorea, 1986), and judgement of the sharpness of edges (Westheimer, 1991).

The same principle generally applies to colour vision. It was shown early that chromatic discrimination deteriorates at short exposures (Brückner \& Kirsch, 1912); and under most stimulus conditions the rule has been found to hold in later studies of wavelength discrimination and purity discrimination (Siegel, 1965; Regan \& Tyler, 1971; Smith, Bowen \& Pokorny, 1984; Uchikawa

*Department of Experimental Psychology, University of Cambridge, Downing Street, Cambridge CB2 3EB, England.

†Abteilung für Sinnes- und Neurophysiologie, Institut für Arbeitsphysiologie an der Universität Dortmund, Ardeystraße 67, D-4600 Dortmund 1, Fed. Rep. Germany.

$\ddagger$ To whom all correspondence should be addressed.
\& Ikeda, 1987; Scase \& Foster, 1988). Data that obey the rule are represented by the open symbols in Fig. 2: the threshold for wavelength discrimination at $500 \mathrm{~nm}$ increases monotonically as the target duration is reduced from 1000 to $1.4 \mathrm{msec}$. Yet the solid symbols in the same figure show a striking counter-example to the rule. When the standard wavelength is $460 \mathrm{~nm}$ rather than $500 \mathrm{~nm}$, the function is almost a mirror-image of that found at $500 \mathrm{~nm}$ : sensitivity deteriorates from a threshold value of $<1 \mathrm{~nm}$ at $2 \mathrm{msec}$ to a value close to $6 \mathrm{~nm}$ at $100 \mathrm{msec}$. One of the two observers of Uchikawa and Ikeda (1968) exhibited an anomaly of this kind in the region of $460 \mathrm{~nm}$ : thresholds were convincingly lower for $8-\mathrm{msec}$, 100 -td fields than for 500-msec, 100-td fields (their Figs 1 and 2). We here examine in detail this short-wave anomaly of chromatic discrimination. We have used a criterion-free psychophysical method to measure wavelength thresholds, in order to exclude the possibility that the anomaly arises because the observer adopts different criteria when discriminating brief flashes and when discriminating long-lasting flashes.

\section{METHODS}

\section{Apparatus}

A bipartite circular field, 2 deg in dia and horizontally divided, was produced by a two-channel Maxwellianview optical system. The light source was a $50 \mathrm{~W}$ tungsten-halogen lamp run from a stabilized supply. The 
optical system was under computer control and incorporated a matched pair of high-resolution monochromators (Bentham Instruments, M300E). Integral stepping motors allowed the centre wavelengths of the monochromators to be changed rapidly in steps of $0.05 \mathrm{~nm}$. The full bandwidths at half height were $3,6 \mathrm{~nm}$. Neutral wedges in each beam allowed luminance to be adjusted in steps of approx. $0.01 \log _{10}$ unit.

The two stimulus beams were combined at a thin mirror, the hevelled edge of which passed horizontally through the optical centres of the beams: the lower half of one beam was reflected at the mirror while the upper half of the second beam passed above the mirror, so that the edge of the mirror formed the horizontal division of the bipartite stimulus field. The combined beams were then focused on a circular $2-\mathrm{mm}$ stop, and a relay of two further lenses was used to form a slightly reduced $(1.6-\mathrm{mm})$ aerial image of the stop in the plane of the subject's pupil. (Such a relay was used as early as 1916 by Ferree and Rand.) An aperture stop in the common beam defined the outer diameter of the circular field and was conjugate with the edge of the mirror. The observer's fixation was guided by a diamond array of four small, dim, white lights, arranged symmetrically about the stimulus field; this array was formed by dissecting out four individual fibres from a fibre-optic cable connected to a tungsten halogen source.

The duration of the flashes was controlled by a pair of electromagnetic shutters, which were placed in the common beam close to the 2-mm stop: energizing one shutter drove a blade out of the beam and energizing the second shutter drove a blade into the beam. The temporal waveforms of the stimuli were regularly monitored by means of a photodiode and a storage oscilloscope. The full rise-time of the flash was $0.75 \mathrm{msec}$ and the fall-time was $1 \mathrm{msec}$. The nominal durations that we give for our flashes are the durations of the rectangular flashes that would contain the same energy; these corrections become significant only at the shortest durations.

Radiometric calibrations were made with a PIN10 photodiode, which had been calibrated spectrally and absolutely by the National Physical Laboratory, Teddington, England.

\section{Procedure}

Wavelength-discrimination thresholds were measured by the two-alternative temporal forced-choice method of Mollon and Cavonius (1987). The method is adapted from procedures that are customarily used to measure auditory frequency discrimination (Moore, Glasberg \& Shailer, 1984). The upper half-field was fixed in wavelength and luminance throughout the experiment,

\footnotetext{
*In some previous studies of wavelength discrimination, preliminary matches of brightness have been obtained at $10 \mathrm{~nm}$ intervals, and a continuous luminosity function has then been obtained by interpolation. Our reason for making matches at intervals of $1 \mathrm{~nm}$ is that the carotenoid-like absorbance spectrum of the macular pigment imposes rapid changes on the luminosity function in the short-wave region, and these variations are especially marked when the stimuli are of very narrow spectral bandwidth.
}
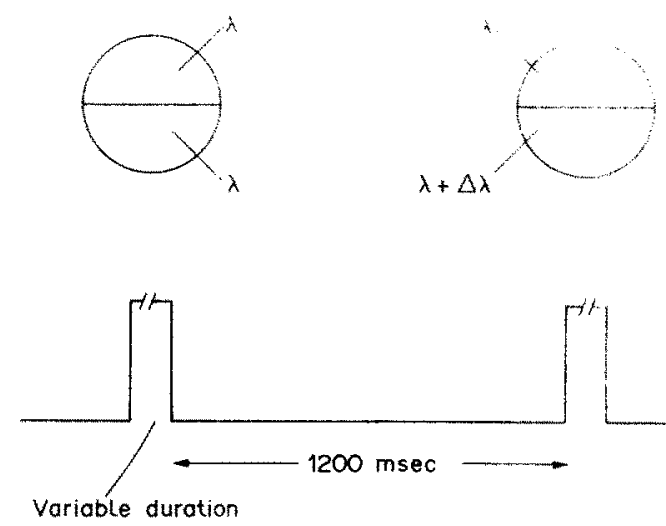

FIGURE 1. Spatial (above) and temporal (below) arrangement of the stimuli for forced-choice measurements of wavelength discrimination. The diagram shows a trial on which a wavelength difference was present on the second of the two presentations; on the other half of trials, chosen at random, the wavelength difference occurred on the first presentation.

whereas the lower half was variable. Each trial consisted of two presentations, separated by $1200 \mathrm{msec}$ (Fig. 1). On one of the two presentations, randomly chosen, the wavelength $(\lambda)$ of the lower half-field was identical to that of the upper half, while on the other its wavelength was $\lambda+\Delta \lambda$. Only positive values of $\Delta \lambda$ were used, and the observer was required to report (by pushing one of two buttons) whether the fields differed on the first or on the second presentation. The correctness of the response was indicated by auditory feedback after each trial. Individual trials were separated by a dark interval of approx. $6 \mathrm{sec}$. The initial value of $\Delta \lambda$ was $3 \mathrm{~nm}$. After two correct responses the value of $\Delta \lambda$ was reduced by $0.097 \mathrm{log}$ unit and after each incorrect response it was increased by $0.097 \mathrm{log}$ unit. Such a staircase tracks the $71 \%$ correct point (Levitt, 1971). The experimental sequence ended when the staircase had reversed 16 times, and the average of the last 10 reversal positions of each run was taken as the threshold. Each run was preceded by 2 min of dark adaptation.

One obstacle to the use of forced choice (or other performance measures) in wavelength discrimination has been the difficulty of ensuring that the observer responds on the basis of hue, and not on the basis of brightness (König \& Dieterici, 1984; Laurens \& Hamilton, 1923). To circumvent this problem we required the observer to make, immediately before each threshold determination, a series of brightness matches between the variable and standard fields. For the first match, the fields were identical in wavelength; the wavelength of the variable field was then increased in increments of $1 \mathrm{~nm}$ until brightness matches had been made at each of 24 wavelengths. These matches were stored and, during the subsequent threshold measurement, the computer automatically equated the brightness of the variable field to that of the standard whenever the wavelength of the variable was changed. Linear interpolation was used to obtain values between those stored for integer values of wavelength.*

To avoid auditory cues from the stepping motors of the monochromators, large random excursions were 
made before both presentations of a given trial. To climinate the effects of backlash in the monochromators, the final wavelength setting was always approached from the same direction; measurements made at the $\mathrm{He}-\mathrm{Ne}$ line showed that it was possible to reset the centre wavelength to within $0.05 \mathrm{~nm}$.

\section{Observers}

The authors served as observers. CRC (male, aged 55) and JDM (male, aged 44) exhibit normal colour vision on the Nagel anomaloscope and the FarnsworthMunsell 100-hue test. SA (female, aged 28) also exhibits normal performance on these tests, but is thought to be a protan carrier.

\section{RESULTS AND DISCUSSION}

\section{Effects of stimulus duration at 500 and $460 \mathrm{~nm}$}

Figure 2 contrasts the opposite ways in which stimulus duration affects wavelength discrimination at $500 \mathrm{~nm}$ and at $460 \mathrm{~nm}$. The retinal illuminance was always $100 \mathrm{td}$ and there were eleven different stimulus durations in the range $1.4 \mathrm{msec}$ to $1 \mathrm{sec}$. Within any one experimental session, the eleven durations were tested in random order at one wavelength. Four such series of measurements were made for each of the two standard wavelengths.

For $500-\mathrm{nm}$ stimuli lasting $1 \mathrm{sec}$, the threshold for wavelength discrimination has the very low value of $0.33 \mathrm{~nm}$ (rightmost open symbol in Fig. 2). As duration is reduced, the threshold rises monotonically and at the shortest duration it reaches a value of over $4 \mathrm{~nm}$. Such a function could be expected from the results obtained at $500 \mathrm{~nm}$ by Uchikawa and Ikeda (1987), who used stimulus durations of 8 and $500 \mathrm{msec}$, and from the results of Scase and Foster (1988), who used stimulus durations of 3 and $1000 \mathrm{msec}$. The absolute values of our thresholds are lower than obtained in these previous studies: this is likely to be because we used a forcedchoice procedure with feedback, because we used highresolution monochromators that allowed steps as small as $0.05 \mathrm{~nm}$, and because we required the observers only to detect the presence of a difference, and not its direction. Uchikawa and Ikeda used a method of adjustment; Scase and Foster used a forced-choice procedure, but did not give feedback.

If the experimental conditions are unchanged, but the standard wavelength is simply shifted by $40 \mathrm{~nm}$ to $460 \mathrm{~nm}$, the effect of target duration is remarkably different (solid symbols, Fig. 2). Performance is optimal at a stimulus duration of only $1.4 \mathrm{msec}$. At this duration the threshold is $<1 \mathrm{~nm}$. As the duration of the stimulus increases, discrimination deteriorates, and at $100 \mathrm{msec}$ the threshold reaches nearly $6 \mathrm{~nm}$. The slight recovery at $1000 \mathrm{msec}$ may be due to the entry of additional factors, such as a (relatively slow) adaptational process or the opportunity for multiple looks; in Figs 2-4 we show the $1000-\mathrm{msec}$ thresholds for reference as isolated data points at the right, but we are concerned in this paper chiefly to explain the paradoxical behaviour of the 460-nm threshold for durations below $100 \mathrm{msec}$.

Interaction of duration and retinal illuminance at $460 \mathrm{~nm}$

Figures 3 and 4 show thresholds for wavelength discrimination at $460 \mathrm{~nm}$ as a function of stimulus duration. The parameter is retinal illuminance. Each threshold was measured in four separate series of runs. Within each series, different illuminances and durations were tested in random order.

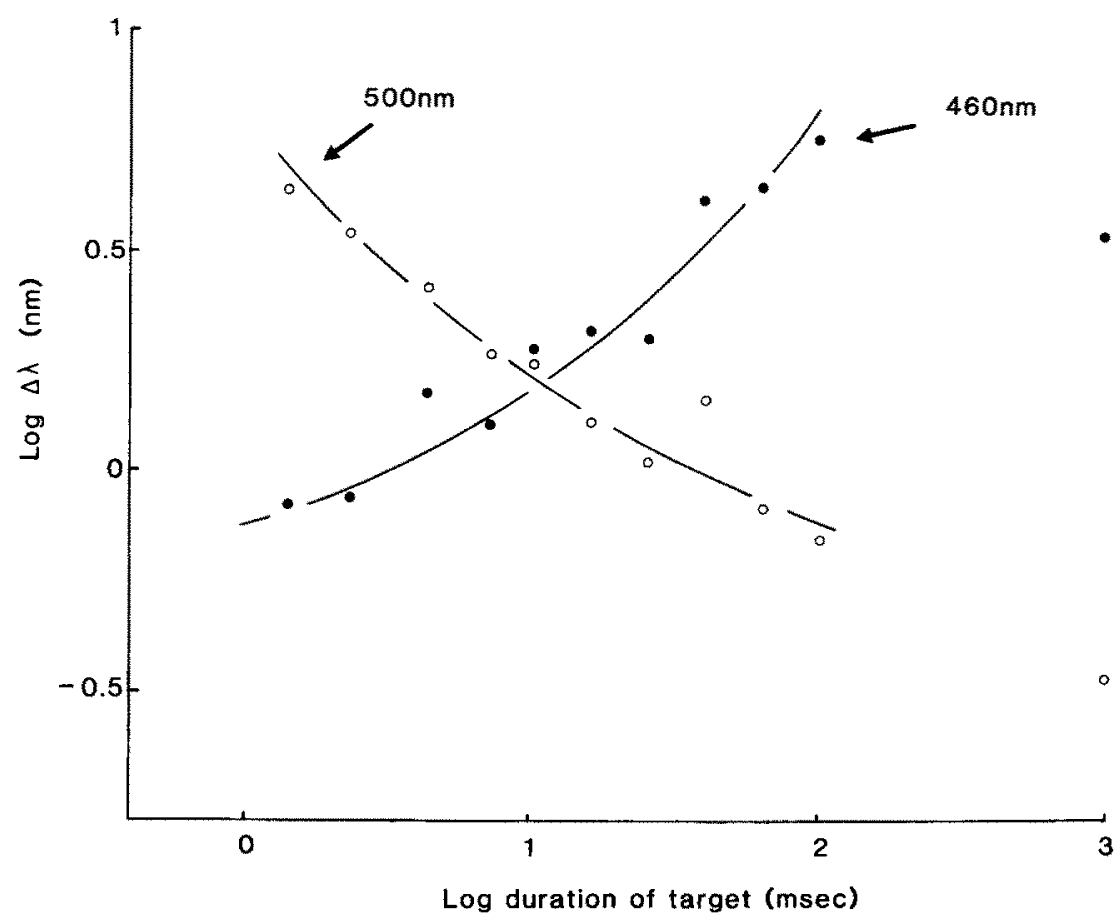

FIGURE 2. Wavelength discrimination as a function of stimulus duration. The ordinate shows the amount by which the wavelength of the lower half of a 2-deg bipartite field must be increased if it is to be discriminated from the upper half at a criterion level of performance. The wavelength of the upper (standard) half-field was either $460 \mathrm{~nm}$ (solid symbols) or $500 \mathrm{~mm}$ (open symbols). The lines drawn through the data for the range $1-100 \mathrm{msec}$ are fitted by eye. 


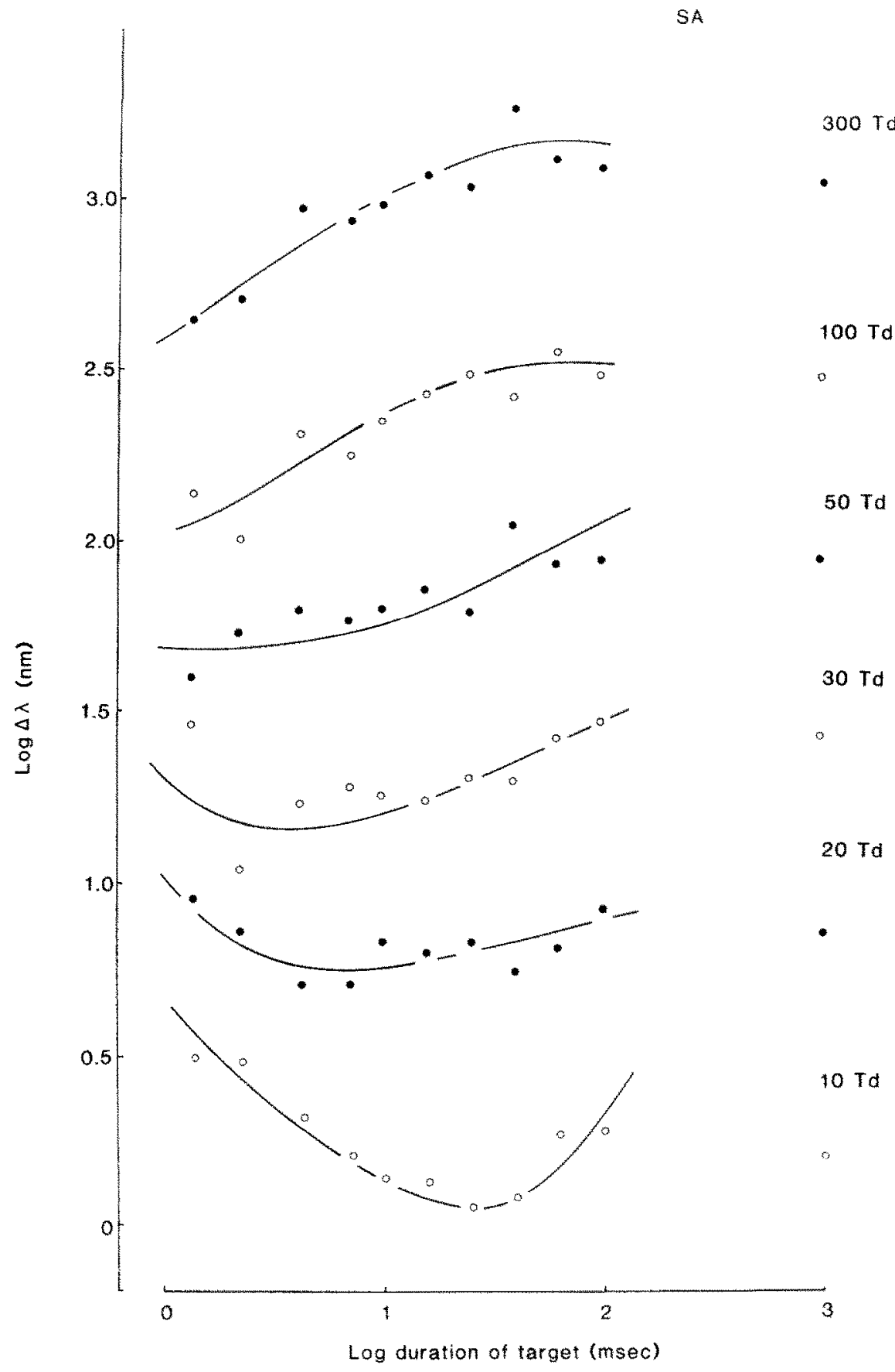

FIGURE 3. Wavelength discrimination for observer SA as a function of stimulus duration for retinal illuminances in the range $10-300 \mathrm{td}$. The standard wavelength was $460 \mathrm{~nm}$. The data at $10 \mathrm{td}$ are placed correctly on the ordinate; successive sets of data are displaced upwards by $0.5 \mathrm{log}$ unit for clarity. The lines drawn through the data for the range $1-100 \mathrm{msec}$ are fitted by eye.

Although the results shown in Figs 3 and 4 may appear complex, very similar patterns are exhibited by the two subjects: at the highest retinal illuminances, performance is best at the shortest durations, whereas at lower illuminances the optimal performance shifts to intermediate durations. Some recovery of sensitivity often occurs when the stimulus duration is extended from 100 to $1000 \mathrm{msec}$ (rightmost data points); the recovery effect is more marked for subject IDM. (Possible reasons for the recovery have been given above.)
Wavelength discrimination as a function of retinal illuminance

To understand the interaction of duration and illuminance seen in Figs 3 and 4, we should first consider another anomaly of wavelength discrimination that occurs in the short-wavelength region of the spectrum. In their classical study of wavelength discrimination, using a performance measure, Köntg and Dieterici (1884) found that sensitivity at $450 \mathrm{~nm}$ was better for a dim stimulus than for a brighter one. A similar result can be 


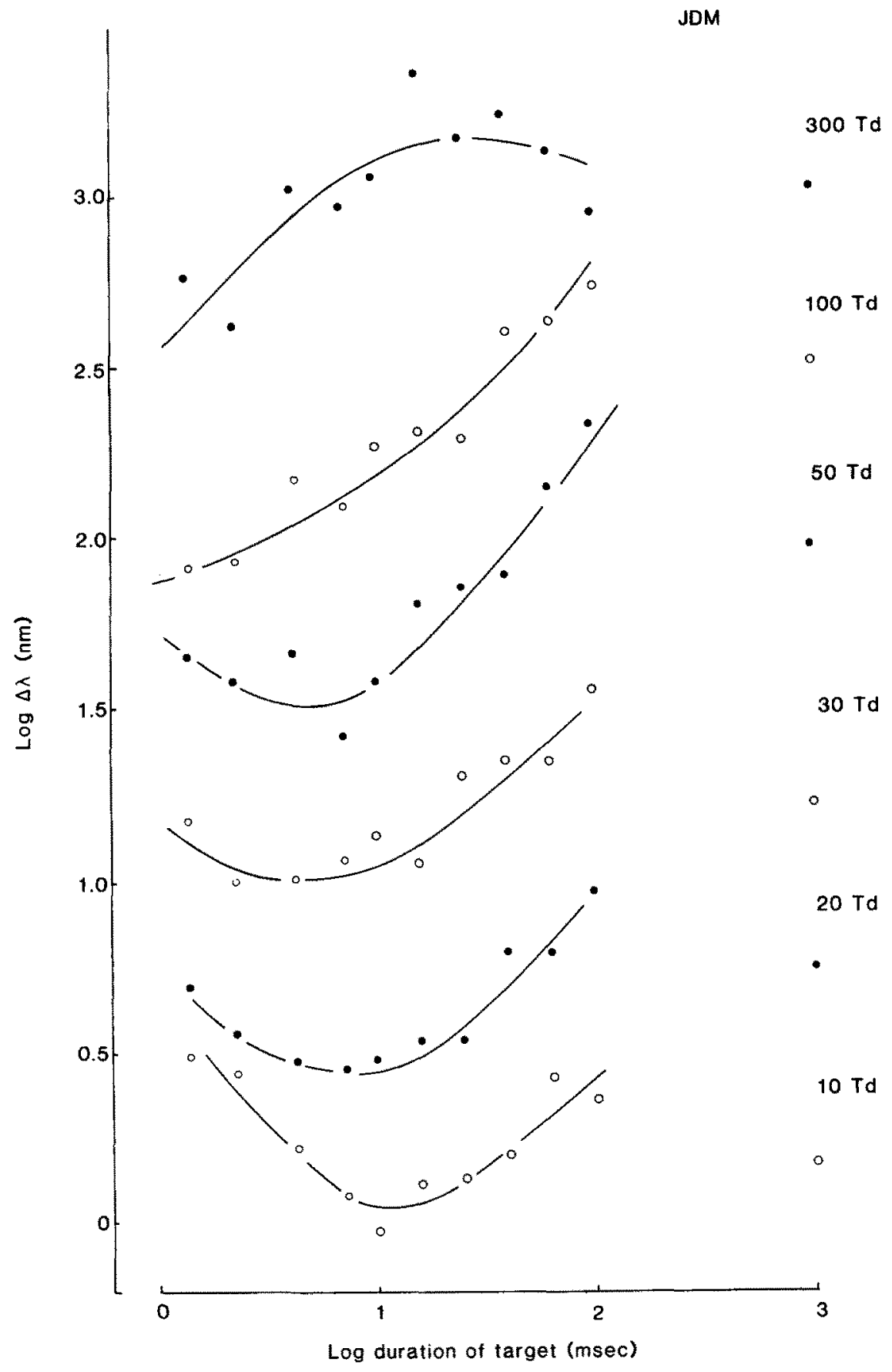

FIGURE 4. Wavelength discrimination at $460 \mathrm{~nm}$ for observer JDM, plotted as in Fig. 3.

seen in the data of Haase (1934). The anomaly was re-discovered by McCree (1960), who studied it for stimulus fields of varying size.

Figure 5 shows the effect under our experimental conditions. The standard wavelength was $460 \mathrm{~nm}$. The duration of the target was always $1000 \mathrm{msec}$ and different retinal illuminances were tested in random order. All three observers show a minimum threshold in the region of $1 \mathrm{td}$ (for JDM and SA the threshold is as low as $0.5 \mathrm{~nm}$ ), whereas above 100 td the thresholds are nearly ten times greater. The observers reported that the stimuli appeared a desaturated blue at the highest troland

*This useful term avoids the ambiguity of referring to a maximum in a wavelength-discrimination function. It has an established usage in population biology. values, whereas richly saturated violets, blues and greens were seen at lower illuminances.

A peak in the foveal wavelength-discrimination curve at $460 \mathrm{~nm}$ has often been reported (e.g. Wright \& Pitt, 1935). Following Weale (1951), we refer to this peak as the "short-wave pessimum".* In the curves published in the literature, the height of the pessimum varies greatly, and sometimes it is completely absent. The functions of Fig. 5 imply that stimulus luminance is a primary factor in determining whether the pessimum will be present.

\section{Wavelength discrimination as a function of stimulus energy}

In the Introduction, we cited a number of instances in which sensory performance varies with the duration of 


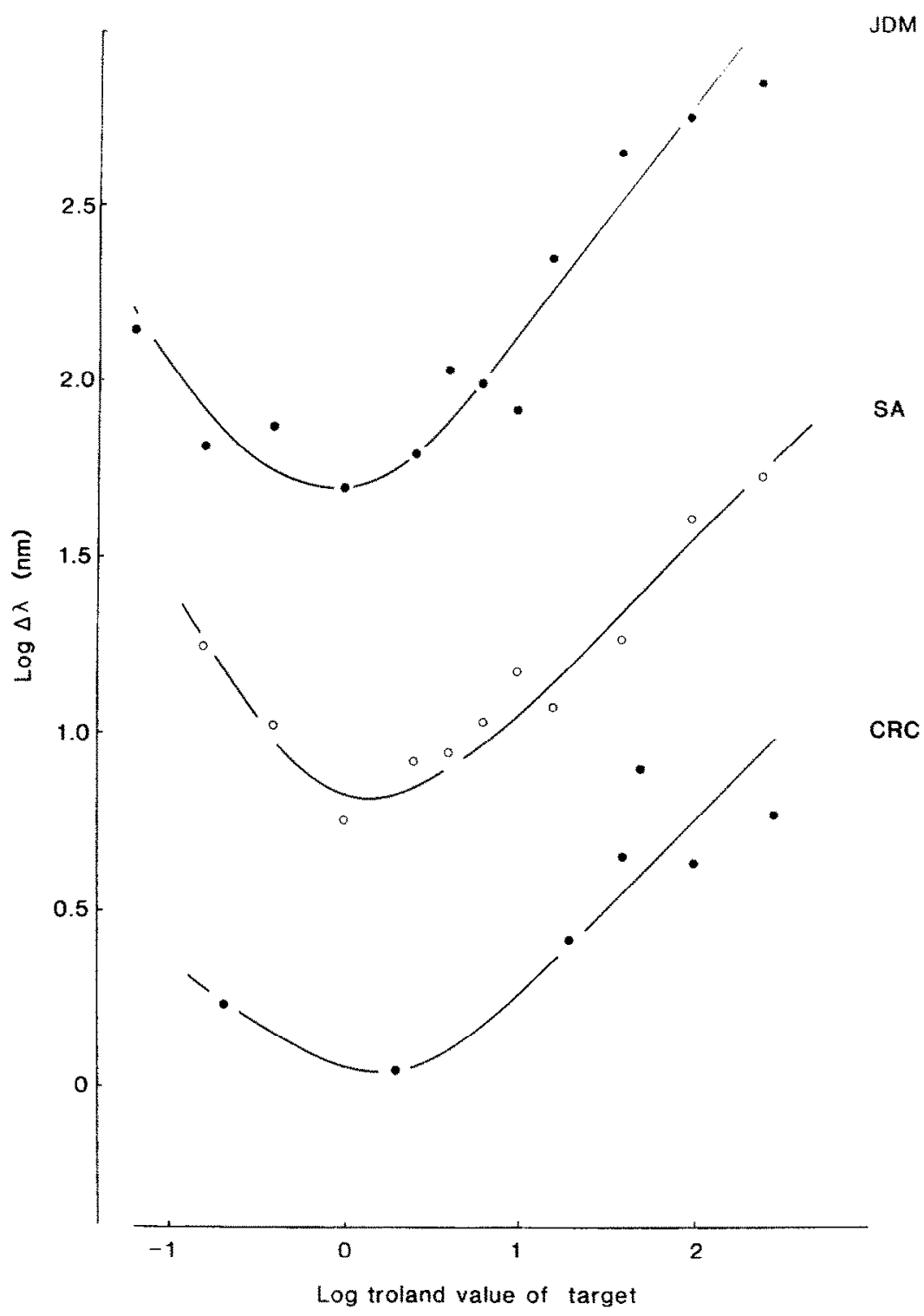

FIGURE 5. Wavelength discrimination as a function of retinal illuminance, for a stimulus duration of 1000 msec. Data for CRC are correctly placed on the ordinate; those of SA and JDM are raised by 1.0 and 2.0 log unit respectively.

the stimulus. It is very unlikely that a common explanation holds for all the examples given there. Nevertheless, in the case of many visual functions, the effect of reducing duration is best regarded as the secondary result of reducing stimulus energy. The visual system always exhibits temporal integration, although the critical duration (the limit of integration) varies for different channels and depends on factors such as adaptation (e.g. Kahneman \& Norman, 1964; Uetski \& Ikeda, 1971; Krauskopf \& Mollon, 1971). It is rare to find a case where variation of duration below $100 \mathrm{msec}$ has an effect on visual sensitivity that is truly temporal, rather than being the consequence of a reduction in energy.*

The results of Fig. 5 show that the threshold for wavelength discrimination at $460 \mathrm{~nm}$ is a non-monotonic function of retinal illuminance when the flashes are relatively long-lasting. So we are led to ask whether the complex results of Figs 3 and 4 can be understood by

*An exception is the study of sharpness judgements by Westheimer (1991). supposing that the threshold is a non-monotonic function of stimulus energy. In Fig. 6, therefore, the data sets for different durations are replotted as a function of the product of duration and illuminance, i.e. as a function of stimulus energy. Only data for durations up to $100 \mathrm{msec}$ are shown, since it is very unlikely that temporal integration of energy extends to $1000 \mathrm{msec}$ and since additional factors may come into play for the longer flashes.

To a first approximation, the data sets for different troland values fall on a single function when plotted against energy in Fig. 6. The solid line in each panel of the figure represent the best-fitting third-order polynomial for that subject's data. These functions have a similar form for the two subjects and both show a minimum threshold near $2 \mathrm{log}$ msec-td. The values of $R^{2}$ are 0.85 for JDM and 0.74 for SA (allowing a higherorder polynomial fit only marginally changes the variance accounted for). By comparison, if the data of Figs 3 and 4 are plotted either against log duration alone or against $\log$ td value alone, and the same analysis is 

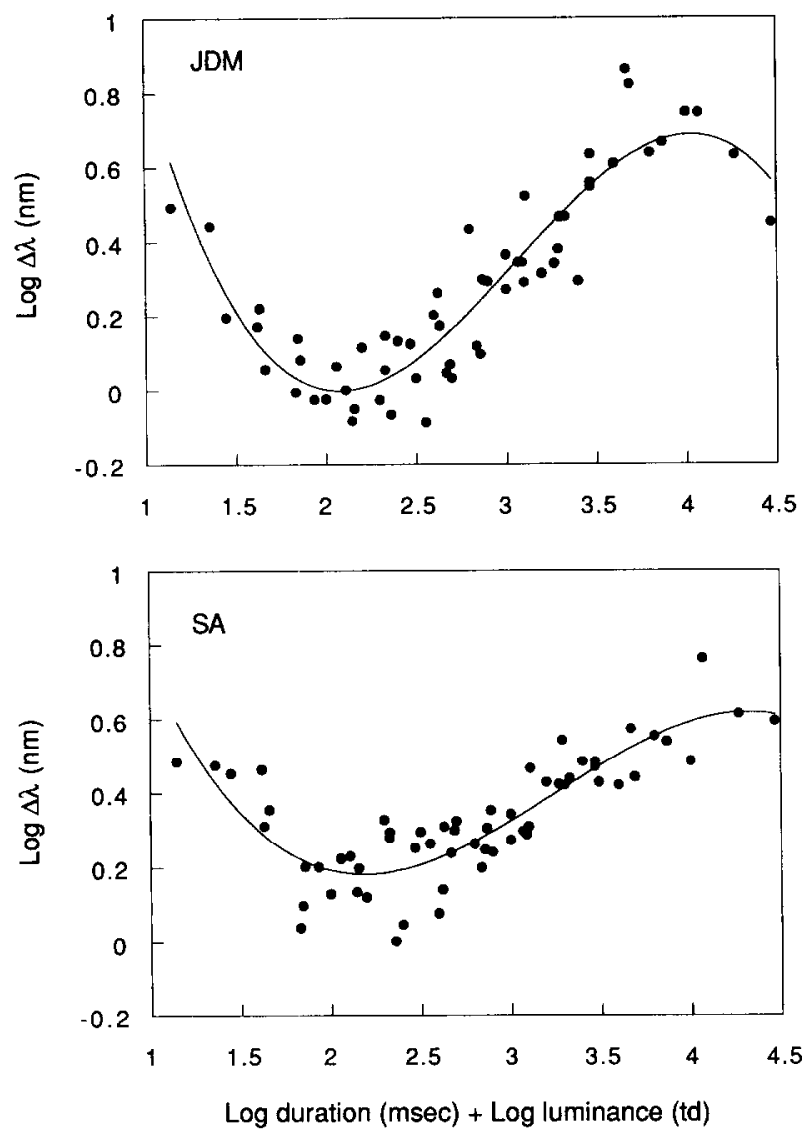

FIGURE 6. Wavelength discrimination as a function of stimulus energy (the product of stimulus duration and retinal illuminance). The data are replotted from Figs 3 and 4 . The solid lines represent best-fitting third-order polynomial functions.

performed, then the values of $R^{2}$ are 0.41 (duration) and 0.23 (td value) for JDM and 0.17 and 0.22 for SA. So we may conclude that the major factor determining hue discrimination at $460 \mathrm{~nm}$ is stimulus energy rather than either duration or retinal illuminance per se.

But this is a very unusual example of Bloch's Law. In almost all the classical cases, sensory discrimination is a positive and monotonic function of the product of intensity and time. Here we find that performance is optimal at an intermediate value of energy. Why should this be the case, and why is such behaviour seen only at short wavelengths?

\section{Isolation of the short-wave system at $460 \mathrm{~nm}$}

Throughout much of the visible spectrum, wavelength discrimination could in principle depend on a comparison of signals from the middle-wave (M) and long-wave (L) cones, since the ratio $\left(S_{\mathrm{M}} / S_{\mathrm{L}}\right)$ of the sensitivities of $M$ and $L$ cones varies continuously over most of the spectrum. However, the spectral region near $460 \mathrm{~nm}$ has a special status: it is the region where the ratio $S_{\mathrm{M}} / S_{\mathrm{L}}$ passes through a flattened maximum (see Estévez \& Cavonius, 1977, Fig. 5; or Boynton, 1979, Fig. 8.3; or Mollon \& Estévez, 1988, Fig. 5). Thus it is a region in which the rate of change of $S_{\mathrm{M}} / S_{\mathrm{L}}$ with wavelength slows to zero, and where a large value of $\Delta \lambda$ is therefore needed before the difference in $S_{\mathrm{M}} / S_{\mathrm{L}}$ is large enough to support hue discrimination.
That $S_{\mathrm{M}} / S_{\mathrm{L}}$ is maximal near $460 \mathrm{~nm}$ is a truth not yet universally acknowledged. Its acceptance is hindered by the unhappy use of the term "red-green system" to refer to the neural channel that extracts the ratio of the $M$ and L signals. For $460 \mathrm{~nm}$ is on the reddish side of unique blue and is not intuitively the wavelength that maximally polarizes a "red-green system" in the greenish direction (Mollon \& Cavonius, 1987). Three lines of evidence may be cited to show that the maximal value of $S_{\mathrm{M}} / S_{\mathrm{L}}$ does lie close to $460 \mathrm{~nm}$ :

(i) A tritanope, or a normal observer viewing under tritanopic conditions, can match all wavelengths with a positive mixture of two primaries, 460 and $650 \mathrm{~nm}$ (Fig. 1 in Willmer \& Wright, 1945; Table II in Wright, 1952). There cannot be a wavelength other than $460 \mathrm{~nm}$ that produces a higher value of $S_{\mathrm{M}} / S_{\mathrm{L}}$, provided only that we allow that the ratio is greater at $460 \mathrm{~nm}$ than at the red primary.

(ii) Using a modified form of Stiles' field-sensitivity method to isolate the cone mechanisms $\pi_{4}$ and $\pi_{5}$ (Estévez \& Cavonius, 1977) showed that the ratio $\pi_{4} / \pi_{5}$ was maximal near $460 \mathrm{~nm}$. (By using auxiliary fields, Estévez and Cavonius probably achieved better isolation in this spectral region than Stiles did.)

(iii) Nunn, Schnapf and Baylor (1985) measured the action spectra for a criterion change in the membrane current of macaque cones: the values for the ratio $S_{\mathrm{M}} / S_{\mathrm{L}}$, obtained in this very direct way, exhibit a maximum at $460 \mathrm{~nm}$.

This then is why tritanopes, and normal observers under tritan conditions of viewing, show a disproportionate loss of hue discrimination at $460 \mathrm{~nm}$ (Wright, 1952; Willmer \& Wright, 1945; Cavonius \& Estévez, 1978; Kaiser \& Boynton, 1985): as the maximum is approached from lower or higher wavelengths, the rate of change with wavelength of $S_{\mathrm{M}} / S_{\mathrm{L}}$ slows down to zero and the subject requires a large increment in wavelength in order to use this ratio to discriminate hue. Post-receptoral adaptation at an opponent site probably provides an additional reason why the ratio $S_{\mathrm{M}} / S_{\mathrm{L}}$ contributes little to wavelength discrimination at $460 \mathrm{~nm}$, since the channel that extracts this ratio must be maximally polarized near $460 \mathrm{~nm}$. From analyses of chromaticity discrimination, Le Grand (1949), Friele (1961) and Boynton and Kambe (1980) have argued that postreceptoral sensitivity is reduced when $S_{\mathrm{M}} / S_{\mathrm{L}}$ becomes very small or very large.

We can conclude that the present experiments at $460 \mathrm{~nm}$ oblige the observer to rely (except possibly at the highest energy levels) on a post-receptoral channel that compares the signal of the short-wave cones with some combination of the signals of the middle- and long-wave cones. This channel is the phylogenetically older of the two main subsystems of primate colour vision (Mollon \& Jordan, 1988/1989). It was explicitly in order to isolate such a channel that we chose to work at $460 \mathrm{~nm}$.

The nature of the saturation of the short-wave system

The data of Fig. 6 are most naturally explained by response saturation. We must suppose that the discrimi- 
nations are mediated by a channel that is saturated by brief flashes of luminous energy greater than $10^{8}$ quanta $\mathrm{deg}^{2}$. Some recovery from saturation may occur if the stimulus is prolonged from 100 to $1000 \mathrm{msec}$, but such a recovery is far from complete, as is shown by the effects of troland value on the discrimination of 1000 msec flashes (Fig. 5).

What is the site at which the saturation occurs? It has commonly been supposed that the short-wave cones saturate, hut the evidence is not conclusive. Recording the electroretinogram from macaques, Norren and Padmos (1973) found that the $b$-wave of the isolated shortwave system grew more slowly than did that for the other cone systems, and saturated at a low response voltage. However, both these features of the ERG could reflect merely the paucity of short-wave cones. Sawusch. Pokorny and Smith (1987) found that the ERG of human short-wavelength cones saturated only above $1000 \mathrm{td}$ (at $460 \mathrm{~nm}$ ). The illuminances used in obtaining the data of our Figs 2-5 were in a range where Sawusch, Pokorny and Smith found the short-wave response to be changing rapidly with troland value. Direct measurements of macaque cones by the suction-electrode technique (Schnapf, Nunn, Meister \& Baylor, 1990) suggest little difference between cone types in their dynamic range. "Blue cone" monochromats offer psychophysical evidence against saturation of the short-wave cones: their acuity continues to improve in the photopic region and does not deteriorate at high luminance levels in the way that the acuity of rod monochromats does (Blackwell \& Blackwell, 1961).

In the case of normal observers, saturation of the psychophysically-defined short-wave mechanism was shown by Mollon and Polden (1977a): when 435-nm targets were presented on $445-\mathrm{nm}$ backgrounds in the presence of a fixed yellow auxiliary field, the threshold vs radiance function rose more steeply than predicted by Weber's Law. But it soon became clear that this psychophysical saturation can be partly or completely eliminated if the radiance of the "auxiliary" long-wave field is increased, so as to yield a composite field that appears achromatic (Mollon \& Polden, 1977b; Stromeyer, Kronauer \& Madsen, 1978; Polden \& Mollon, 1980): under these conditions of combinative euchromatopsia the threshold for the short-wave target is lower on the composite field than it is when only the short-wave component of the ficld is present. The higher the radiance of the long-wave field, the higher must be the radiance of the short-wave field if thresholds are to rise above the Weber line (Polden \& Mollon, 1980, Figs 13 and 14$)$.

In sum, there is little evidence that the short-wave cones per se exhibit saturation at illuminances as low as $100 \mathrm{td}$, whereas there is good evidence for a compressive non-linearity at a chromatically-opponent site through which the short-wave signals pass. Yet the latter site does not behave in the way that might be expected of a mechanism designed for colour recognition: if a channel is designed to recognize the reflection spectra of objects independently of the level of illumination, then ideally it should respond to the ratio of the quantum catches of different classes of cone, and should not be sensitive to the absolute level of stimulation. To explain the energydependence seen in the present results, we must suppose that the signal reaching the opponent site from the short-wave cones grows more rapidly with energy than do the signals of other cones; or else we must suppose that the response of the opponent site is some function of the absolute difference of the quantum catches and not strictly of their ratio.

\section{The varieties of tritanopia}

Over a large range of stimulus conditions we can rely on the signals of our long- and middle-wave cones to sustain our visual discriminations. By comparison, there is a much more constrained range of conditions over which we obtain discriminable signals from the shortwave cones; and several stimulus parameters (e.g. luminance, duration, size, retinal locus) can be manipulated so as to make the normal observer behave like a tritanope. Some writers have sought a single factor to account for these functional tritanopias (Farnsworth, 1956) and indeed a factor underlying several of them may be the sparseness of short-wave cones (Sperling. 1980; Dartnall, Bowmaker \& Mollon, 1983); but it may be useful to distinguish at least four forms of functional tritanopia:

(i) The complete tritanopia that arises from the absence of short-wave cones in the 20-minarc centre of the foveola (Wald, 1967; Williams, MacLeod \& Hayhoe, 1981a).

(ii) The high detection threshold that results from the sparseness of short-wave cones in the remainder of the retina (Williams, MacLeod \& Hayhoe, 1981b). The absolute sensitivity of individual short-wave cones may be comparable to that of other cones, but spatial integration cannot extend the psychophysically-measured sensitivity to low energies as it does for other cones.

(iii) The spatial imprecision of the short-wave signals when the discriminanda are small juxtaposed fields (Cavonius \& Estévez, 1978).

(iv) The saturation that can occur at a post-receptoral opponent site through which the short-wave signals pass (Polden \& Mollon, 1980).

More than one of these factors constrains the thresholds plotted in Fig. 2. At $500 \mathrm{~nm}$, the short-wave opponent channel is not polarized, but the short-wave cones are 27 times less sensitive to a light of a given troland value than they are to a $460-\mathrm{nm}$ light of the same troland value (Boynton \& Kambe, 1980, Fig. 4). When the duration of a $100-\mathrm{td} 500-\mathrm{nm}$ flash is reduced, we may suppose that the signal of the short-wave cones comes to be limited by the reduction in energy. Thus, in this case. performance in the wavelength-discrimination task is constrained by a shortage of photon absorptions. On the other hand, at $460 \mathrm{~nm}$, which is much closer to the wavelength of peak sensitivity of the short-wave cones, there is no shortage of isomerizations in the cones themselves and discrimination is limited by polarization of an opponent channel. This limitation is reached only 


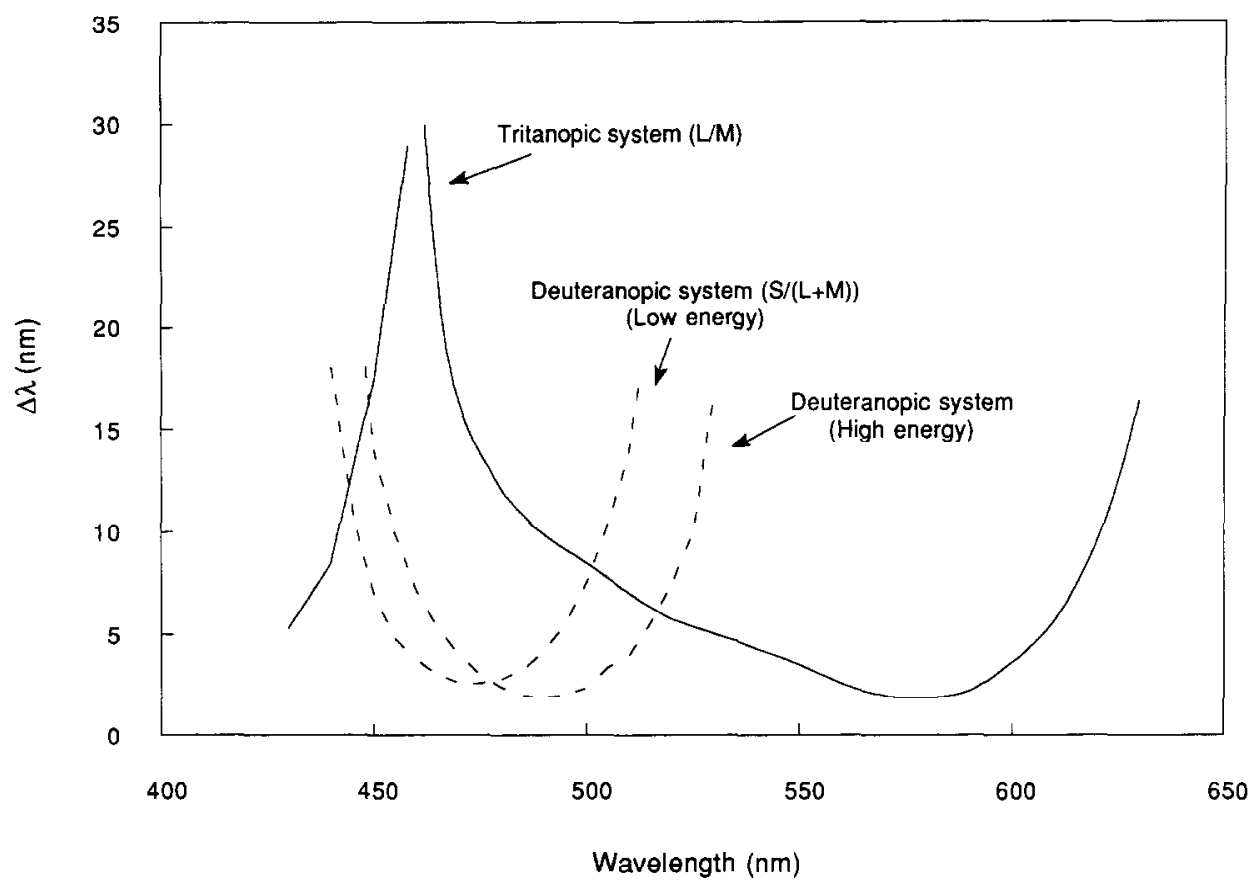

FIGURE 7. A schematic representation of how wavelength discrimination depends on the sensitivities of two underlying subsystems. The phylogenetically newer "tritanopic" subsystem is represented by the solid line and the more ancient "deuteranopic" subsystem is represented by the two dashed curves (which correspond to high and low stimulus energies). Note that the threshold for the younger subsystem becomes very large near $460 \mathrm{~nm}$, where the ratio $S_{\mathrm{M}} / S_{\mathrm{L}}$ passes through a flat maximum. The $\mathrm{U}$-shaped function for the ancient subsystem shifts towards shorter wavelengths as either stimulus duration or stimulus luminance is reduced, thus giving a gain in sensitivity at $460 \mathrm{~nm}$ and a loss of sensitivity at $500 \mathrm{~nm}$ (as seen empirically in Fig. 2). The functions shown here are schematic, but the deuteranopic component is based on the wavelength-discrimination curves given for deuteranopes by Pitt (1944) and by Walraven and Bouman (1966); and the tritanopic component is based on tritanope " $\mathrm{A}$ " in the study of Wright (1952) and the functions obtained by Cavonius and Estévez (1978) for normal observers under tritan conditions of viewing.

when stimulus energy is relatively high, and thus wavelength discrimination in this case improves as stimulus duration decreases.

Figure 7, which is derived from Walraven and Bouman (1966), summarizes our interpretation of how wavelength discrimination is determined in this spectral region (Mollon, Estévez \& Cavonius, 1990). The subject's overall performance depends on two subsystems. The phylogenetically older subsystem compares the short-wave cone signal with some combination of the signals of the long- and middle-wave cones (Mollon \& Jordan, 1988/89), and is here referred to as the deuter-

*Stimulus conditions that exhibit "tachistoscopic tritanopia" (Mollon, 1982) are thus cases where there is not an initially high excitation of the short-wave cones and where short durations therefore force the operating range to lie on the right-hand limb of the deuteranopic component of Fig. 7, inducing a form of the "threshold tritanopia" that Farnsworth (1955) postulated. Such would be the case in the experiment of Weitzman and Kinney (1967), who found that dim yellow stimuli $(560-580 \mathrm{~nm}, 0.17 \mathrm{ft}-\mathrm{L})$ looked white at short exposures; and also in the study of Taylor (1983) who found that normal subjects made tritan errors when the pigment colours of the City University Test (especially plates 7, 8, 9 and 10) were exposed only briefly. But short durations do not invariably induce tritanopia as the present study shows. And what holds for duration, holds mutatis mutandis for illumination level: normal subjects will often behave like tritans when performing reflective colour tests at low illuminations (van Everdingen, Smith \& Pokorny, 1991), but we should expect this simulation of tritanopia to be valid only when the initial excitation of the short-wave cones was weak anopic subsystem (following Walraven \& Bouman). The phylogenetically younger subsystem extracts the ratio $S_{\mathrm{M}} / S_{\mathrm{L}}$ and is here referred to as the tritanopic subsystem. We assume for simplicity that the measured performance of the subject depends on the lower envelope of the sensitivities of the two subsystems.

The sensitivity of the deuteranopic subsystem is represented in Fig. 7 by dashed lines. At both low and high intensities, the function is U-shaped; and we suppose that it is limited on the long-wave side by a shortage of photon absorptions in the short-wave cones and on the short-wave side by saturation of an opponent site. Only for a narrow range of wavelengths is discrimination optimal. As stimulus energy is reduced, the function shifts to the left, so that the optimum lies at shorter wavelengths. Such a shift has been demonstrated empirically in the wavelength-discrimination function of a deuteranope, who retains only the older subsystem of colour vision (Walraven \& Bouman, 1966). It is clear from Fig. 7 that a leftward shift will gencrate the two contrary effects that are seen as duration is reduced in Fig. 2: at $460 \mathrm{~nm}$ sensitivity increases and at $500 \mathrm{~nm}$ sensitivity is lost.*

The solid line in Fig. 7 represents the postulated sensitivity of the phylogenetically newer, tritanopic, subsystem of colour vision. This subsystem remains quite sensitive throughout the spectrum, except near $460 \mathrm{~nm}$, where the rate of change of $S_{\mathrm{M}} / S_{\mathrm{L}}$ goes to zero, as described earlier. The difference in the behaviour of the 
two subsystems may reflect the positions of the three cones: the older subsystem draws its opposed inputs from cones that are well-separated in the spectrum and it is thus presented with a very large range of ratios of quantum catches, but the long- and middle-wave cones overlap extensively in their sensitivities so that the ratio of their quantum catches is never very small or very large, and the opponent site that receives their signals is more immune to saturation.

A decrease in duration and a decrease in area are usually equivalent in their effects on visual sensitivity. The present results show an exception to this principle: a reduction of duration enhances discrimination at $460 \mathrm{~nm}$, whereas Willmer and Wright (1945) and McCree (1960) found that reducing stimulus size impairs wavelength discrimination in this part of the spectrum. One explanation of this discrepancy can be found in factor (iii). Since the short-wave cones are distributed in a sparse matrix, and since the short-wave bipolar cells probably draw their input from more than one cone (Mariani, 1984), the signal of the older colour channel carries with it only an imprecise local sign. Therefore, if the observer is required to discriminate two small and juxtaposed half-fields, his difficulty may arise because the short-wave cone signal cannot be attributed exclusively to one half-field, and not because the short-wave signal is too small or is saturated.

\section{REFERENCES}

Andrews, D. P. (1967). Perception of contour orientation in the central fovea. Part I: Short lines. Vision Research, 7, 975-997.

Blackwell, H. R. \& Blackwell, O. M. (1961). Rod and cone mechanisms in typical and atypical congenital achromatopsia. Vision Research, $1,62-107$.

Boynton, R. M. \& Kambe, N. (1980). Chromatic difference steps of moderate size measured along theoretically critical axes. Color Research and Application, 5, 13.23.

Brückner, A. \& Kirsch, R. (1912). Untersüchungen über die Farbenzeitschwelle. Zeitschrift für Sinnesphysiologie, 46, 229-286.

Cavonius, C. R. \& Estévez, O. (1978), $\pi$-Mechanisms and cone fundamentals. In Armington, J. C., Krauskopf, J. \& Wooten, B. R. (Eds), Visual psychophysics and physiology. New York: Academic Press.

Dartnall, H. J. A., Bowmaker, J. K. \& Mollon, J. D. (1983). Human visual pigments: Microspectrophotometric results from the eyes of seven persons. Proceedings of the Royal Society of London, B, 220, $115-130$.

Estévez, O. \& Cavonius, C. R. (1977). Human color perception and Stiles' $\pi$ mechanisms. Vision Research, 17, 417-422.

van Everdingen, J. A. M., Smith, V. C. \& Pokorny, J. (1991). Sensitivity of tritan screening tests as evaluated in normals at reduced levels of illumination. In Drum, B., Moreland, J. D. \& Serra, A. (Eds), Colour vision deficiencies $X$. Dordrecht: Kluwer.

Farnsworth, D. (1955). Tritanomalous vision as a threshold function. Die Farbe, 4, 185-195.

Ferree, C. E. \& Rand, G. (1916). A substitute for an artificial pupil. Psychological Review, 23, 380-382.

Foley, J. M. \& Tyler, C. W. (1976). Effect of stimulus duration on stereo and vernier displacement thresholds. Perception and Psychophysics, 20, 125-128.

Friele, L. F. C. (1961). Analysis of the Brown and Brown-MacAdam colour discrimination data. Die Farbe, 10, 193-224.

Graham, C. H. \& Kemp, E. H. (1938). Brightness discrimination as a function of the duration of the increment in intensity. Journal af General Physiology, 21, 635-650.
Haase, G. (1934). Bestimmung der Farbtonemfindtichkel des men schlichen Auges bei verschieden Helligkeiten und Sätigungen. Bau eines empfindlichen Farbpyrometers, Annalew dit phrik. Wh 75. 105

Kahneman, D. \& Norman, J. (1964). The time matsuty relation th visual perception as a function of observer' iask. lanmal if Experimental Psychology, 68,215 220.

Kaiser, P. K. \& Boynton, R. M. (1985). Role of the blue mechanism in wavelength discrimination. Vision Restarch. $\quad 5,523529$

König. A. \& Dieterici, C. (1884). Über die Empfindlichkeit des normalen Auges für Wellenlängenunterschiede des Lichtes. Annalen der Physik und Chemie, 22, 579.589.

Krauskopf, 3. \& Mollon, J. D. (1971). The independence of the temporal integration properties of individual chromatic mechanisms in the human eye. Journal of Physiology, London. 279 611623 .

Laurens, H. \& Hamilton, W. F. (1923). The sensibility of the eyc to differences in wavelength. American Joumal of Physiology, 65 $547-568$.

Le Grand, Y. (1949). Les seuils différentiels de couleurs dans la théorie de Young. Revue d'Optique, 28, 262-277.

Leibowitz, H., Mitchell, E. \& Angrist, N. (1954). Exposure duration in the perception of shape. Science, $120,400$.

Levitt, H. (1971). Transformed up-down method in psychoacoustics. Journal of the Acoustical Society of American, 49, 467,477.

Mariani, A. P. (1984). Bipolar cells in monkey retina selective for cone likely to be blue-sensitive. Nature, $308,184186$.

McCree, K. J. (1960). Small field tritanopia and the effects of voluntary fixation. Optica Acta, 7.317-323.

Mollon, J. D. (1982). A taxonomy of tritanopias. Documenta Ophthat mologica Proceedings Series, 33, 87-101. (Colour vision deficiencies $V I$, Verriest $G$. (Ed.). The Hague, Junk.)

Mollon, J. D. \& Cavonius, C. R. (1987). The chromatic anatagonisms of opponent process theory are not the same as those revealed in studies of detection and discrimination. In Verriest. G. (Ed.), Colour vision deficiencies VIII. Dordrecht: Nijhoff/Junk

Mollon. J. D. \& Estévez. O. (1988). Tyndall's paradox of hue discrimination. Journal of the Optical Society of America A, 5 , $151 \% 159$.

Mollon, J. D. \& Jordan, G. (1988/1989). Eine evolutionäre Interpretation des menschlichen Farbensehen. Die Farbe. 35/36, $139-170$

Mollon, J. D. \& Polden, P. G. (1977a). Saturation of a retinal cone mechanism. Nature, 265, 243-246.

Mollon. J. D. \& Polden, P. G. (1977b). Further anomalies of the blue mechanism. Investigative Ophthalmology and Visual Science, It, Suppl., 140.

Mollon, J. D., Estévez, O. \& Cavonius, C. R. (1990). The two systems of colour vision and their roles in wavelength discrimination. In Blakemore, C. (Ed.). Vision: Coding and efficiency. Cambridge: Cambridge University Press.

Moore, B. C. J. (1973). Frequency limens for short-duration tones. Journal of the Acoustical Society of America, 54, 610-619.

Moore. B. C. J., Glasberg, B. R. \& Shailer, M. J. (1984). Frequency and intensity difference limens for harmonics within complex tones. Journal of the Acoustical Society of America, 75, 550-561.

Norren, D. V. Padmos, P. (1973). Human and macaque blue cones studied with electroretinography. Vision Research, 13, $1241 \cdot 1254$.

Nunn, B. J., Schnapf, J. L. \& Baylor, D. A. (1985). The action spectra of rods and red-and green-sensitive cones of the monkey Macaca fascicularis. In Ottoson, D. \& Zeki, S. (Eds), Central and peripheral mechanisms of colour vision. London: Macmillan.

Nyman, G., Laurinen, P. \& Campbell, F. W. (1986). Image processing in human vision: The analysis of spatial phase in peripheral vision. Acta Universitatis Oulu, A 179, 1986, 39-42

Pitt, F. H. G. (1944). The nature of normal trichromatic and dichromatic vision. Proceedings of the Royal Society of London, B. 132. $101-117$.

Polden, P. G. \& Mollon, J. D. (1980), Reversed effect of adapting stimuli on visual sensitivity. Proceedings of the Roval Society of London, B. 210, 235-272 
Regan, D. \& Tyler, C. W. (1971). Temporal summation and its limit for wavelength changes: an analog of Bloch's Law for color vision. Joumal of the Optical Society of America, $A, 61$, $1414-1421$.

Sawusch, M., Pokorny, J. \& Smith, V. C. (1987). Clinical electroretinography for short wavelength sensitive cones. Investigative Ophthaimology and Visual Science, 28, 966-974.

Scase, M. O. \& Foster, D. H. (1988). Anomalous loss in blue-green wavelength discrimination with very brief monochromatic stimuli presented to the normal human eye. Ophthalmic and Physiological Optics, 8, 193-198.

Schnapf, J. L., Nunn, B. J., Meister, M. \& Baylor, D. A. (1990). Visua transduction in cones of the monkey, Macaca fascicularis. Journal of Physiology, London, 427, 681-713.

Siegel, M. H. (1965). Color discrimination as a tunction of exposure time. Journal of the Optical Society of America, 55, 566-568.

Smith, V. C., Bowen, R. W. \& Pokorny, J. (1984). Threshold temporal integration of chromatic stimuli. Vision Research, 24 $653-660$

Sperling, H. G. (1980). Blue receptor distribution in primates from intense light and histochemical studies. In Verriest, G. (Ed.), Colour vision deficiencies $\boldsymbol{V}$. Bristol: Hilger.

Stromeyer, C. F., Kronauer, R. E. \& Madsen, J. C. (1978). Apparent saturation of blue-sensitive cones occurs at a color-opponent stage Science, 202, 217-219.

Taylor, S. P. (1983). A time induced tritan defect. Vision Research, 23 , $745-748$.

Tyler, C. W. \& Gorea, A. (1986). Different encoding mechanisms for phase and contrast. Vision Research, 26, 1073-1082.

Uchikawa, K.\& Ikeda, M. (1987). Color discrimination and appearance of short-duration equal-luminance monochromatic lights. Journal of the Optical Society of America, A, 4, 1097-1103.
Uetsuki, T. \& Ikeda, M, (1971). Adaptation and critical duration for Stiles' $\pi$ mechanisms. Journal of the Optical Society of America, 61 , $821-828$.

Wald, G. (1967). Blue-blindness in the normal fovea. Journal of the Optical Suciety of America, 57, 1289-1301.

Walraven, P. L. \& Bouman, M. A. (1966). Fluctuation theory of colour discrimination of normal trichromats. Vision Research, 6, 567-586.

Weale, R. A. (1951). Hue-discrimination in para-central parts of the human retina measured at different luminance levels. Journal of Physiology, London, 113, 115-122.

Weitzman, D. O. \& Kinney, J. A. (1967), Appearance of color for small, brief, spectral stimuli in the central fovea. Journal of the Optical Society of America, 57, 665-670.

Westheimer, G. (1991). Sharpness discrimination of foveal targets Journal of the Optical Society of America, A, 8, 681-685.

Williams, D. R., Macleod, D. I. A. \& Hayhoe, M. H. (1981a). Foveal tritanopia. Vision Research, 21, 1341-1356.

Williams, D. R., Macleod, D. I. A. \& Hayhoe, M. H. (1981b). Punctate sensitivity of the blue-sensitive mechanism. Vision Research, 21, 1357-1375.

Willmer, E. N. \& Wright, W. D. (1945). Colour sensitivity of the foveal centralis. Nature, 156, 119.

Wright, W. D. (1952). The characteristics of tritanopia. Journal of the Optical Society of America, 42, 509-521

Wright, W. D. \& Pitt, F. G. H. (1935). Hue-discrimination in normal colour-vision. Proceedings of the Physical Society, 46, 459-473.

Acknowledgements-This work was supported by MRC Grant G8807036N and by a travel grant from British German programme for Academic Research Collaboration. We are grateful to Gabriele Jordan for assistance and to Dr M. Webster and Dr C. Stromeyer for comments on the text. 